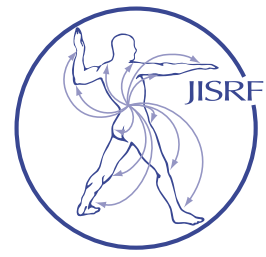

\title{
Understanding lliotibial Band-Sparing Total Hip Arthroplasty: Alternatives to Traditional THA Approaches
}

\author{
Nevins $R^{1}$, Sagers ${ }^{2}$
}

\section{Abstract}

Excellent long term results in total hip arthroplasty (THA) are achievable through a variety of surgical techniques. However, the push for cost savings and higher patient expectations has shifted the focus to improving short term outcomes such as length of stay and in-hospital narcotic requirements. While approximately $87 \%$ of surgeons worldwide continue to prefer traditional posterolateral (PL) or lateral approaches for arthroplasty, [1] alternative approaches that spare the iliotibial band have emerged over the last several years in hopes of improved outcomes. This review explores the iliotibial band-sparing approaches, their advantages and disadvantages, and provides an overview of their published results.

\section{The Iliotibial Band}

The Iliotibial band (ITB) is a group of vertically oriented fibers consisting of a superficial, intermediate and deep layer that converge from their origins at the iliac tubercle, iliac crest and the superior acetabulum and insert on the lateral femoral condyle and tibia. Huang et al, who dissected 40 cadaver specimens and examined the ITB and investing structures at the hip, describe the insertion of the superior fibers of the gluteus maximus as a posterior reinforcement of the ITB. [2] These fibers do not insert at the greater trochanter, but rather join the continuation of the tensor fascia latae and the remaining fibers of the inferior gluteus max- imus to insert at the linea aspera via the lateral intermuscular septum. [ㅁ-4] (FIGURE 1)

Much study and emphasis has more recently been focused on the function of the ITB at the knee, where it functions to create a rigid support pillar to allow for asymmetric standing. [ㄱ-5 $\underline{5}$ However, Inman attempted to qualify and quantify its contribution to standing and gait at the hip as early as 1947. He proposed that the ITB provides nearly half of the torque necessary to keep the pelvis level

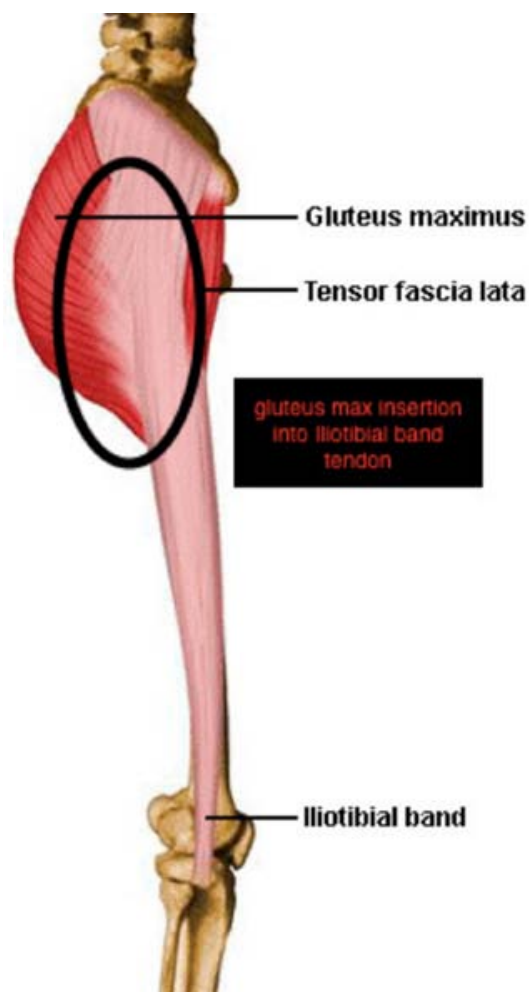

Figure 1: Approximately $75 \%$ of gluteus maximus insertion is into the iliotibial bad. Image courtesy of dept. washington.edu

Keywords: iliotibial band; total hip arthroplasty; minimally invasive

Level of Evidence: AAOS Therapeutic Level V 
and is one of the final restraints to prevent further sagging when the pelvis is allowed to sag unilaterally. []] The result of disrupting this checkrein to hip abduction during single leg stance has been shown in literature evaluating lateral THA approaches, with the incidence of Trendelenburg gait as high as $31 \%$. [7]

\section{Direct Anterior Approach}

The Direct Anterior (DA) Approach is the most popular and studied of the ITB sparing approaches. The intermuscular, internervous interval of the approach, with the plane extending between the tensor fascia latae (TFL) and the sartorius muscles, was originally described in 1881 by Hueter, []] and subsequently modified by Smith-Petersen in 1917. [9] It was not routinely used in THA until 1980 when Light and Keggi published their results, which showed a low complication rate, although average length of stay (LOS) was 12.8 days in their study. [10] Over the last 20 years, renewed interest in the DA approach has been driven by the search for a less invasive approach with faster recovery.

The DA approach can be performed using either specialized tables or a standard operating table, depending on surgeon preference. Specialized tables allow traction boots to be used and may allow for more precise positioning and control of the extremities during the surgery. When using a standard table, a bump may be placed beneath the patient at the level of the anterior superior iliac spine (ASIS) to allow relative extension of the hip to allow for better exposure during femoral preparation. In addition, the table itself can be flexed in a way to allow for additional hip extension if the patient is positioned at the crease of the table.

While placement of the incision may vary slightly with surgeon preference, it generally extends from approximately $3 \mathrm{~cm}$ inferior and $3 \mathrm{~cm}$ lateral to the ASIS, extending distally over the TFL muscle belly. Dissection of the fascial planes of the TFL is carried out to ensure proper muscular intervals and avoid medial dissection near the femoral neurovascular bundle. After separating the TFL from the sartorius, the TFL is retracted laterally, retractors are placed extracapsularly over the superior and inferior femoral neck as well as the lateral edge of the intertrochanteric ridge. (FIGURE 2) The fat overlying the anterior hip capsule is removed, and the attachment of the reflected head of the rectus femoris is released from the anterior acetabulum. Capsulotomy or capsulectomy is then performed and the joint exposed by placing the retractors intracapsularly. The femoral neck cut is made and the head is extricated. The acetabulum is then prepared and the cup placed, often-

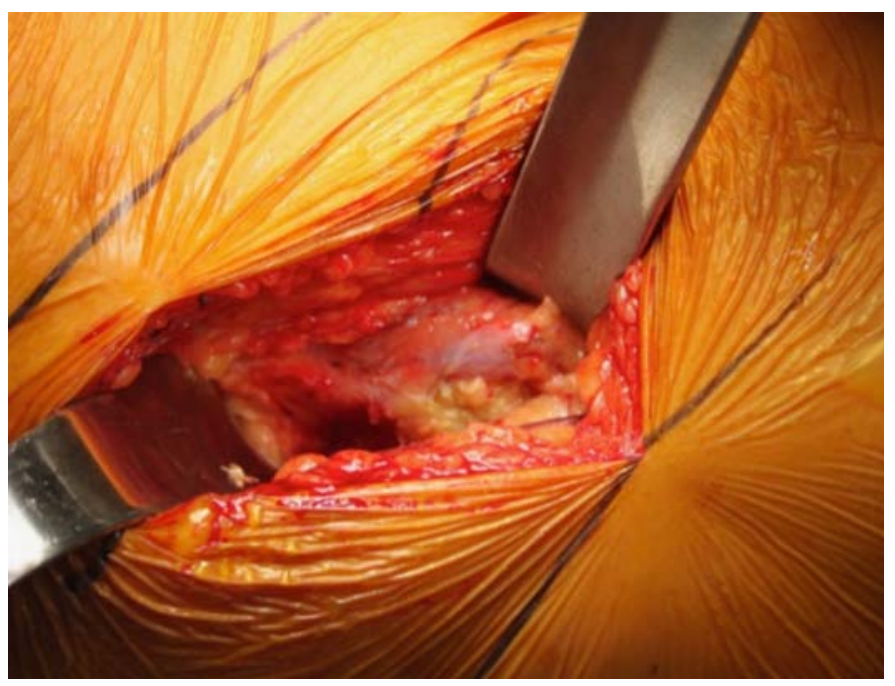

Figure 2: DA Approach superficial dissection is performed between TFL and sartorius. Image courtesy of Dr. Aaron Salyapongse

times under fluoroscopic guidance to ensure proper positioning. The femoral shaft is then presented by extending, external rotating and adducting the hip. Specialized bone hooks and offset broaches have been designed to assist in presenting the femur out of the incision. [11] Early teaching of the technique also described a release of the posterolateral capsule at the greater trochanter to allow for further translation; however, several authors now recommend releasing the conjoined tendon to the level of the obturator internus and, when necessary, the piriformis, to facilitate adequate exposure. [12-14] After femur preparation is complete, trialing, final implantation and stability testing takes place. The wound is closed in the standard fashion. While individual protocols vary, many DA surgeons do not place formal restrictions on mobility for their patients postoperatively.

Outcomes of the DA approach have been favorable, particularly in the early postoperative period. Barrett et al performed a randomized trial comparing DA and PL approaches performed by the same surgeon. The DA patients had less pain, were discharged sooner, and were able to walk greater distances during the first and second postoperative days in comparison to the PL approach patients. However, the longer-term outcomes were equal at 6 months between the two groups. [15] Restrepo et al performed a randomized study comparing the DA and direct lateral (DL) approach and had similar improved short term outcomes in the DA group. [16] Berend performed a large retrospective study comparing the DA and the less-invasive DL approach. The LOS between the groups was not significantly different, but the percentage of patients discharged home, the Harris Hip Scores and Lower Extremity Activity Scores were all significantly higher in the DA vs. the DL group. [17] Several studies support these findings, 
[18-22] while others refute them. [23]

Initially described and advertised as a "tendon sparing" approach (focused on sparing the short external rotators (SER) in comparison to the posterolateral approach), releases of the piriformis and conjoined tendon better facilitate femoral preparation during the DA approach. [12-14] Meneghini performed a cadaveric study comparing muscle damage incurred by DA and PL approaches and reported that release of the piriformis and conjoined tendon was necessary in $50 \%$ of their dissections in order to achieve adequate femoral exposure. [14] This, however, does not appear to compromise the potential for accelerated recovery. Rodriguez et al compared 60 patients that had the DA approach THA using a release of the posterior superior hip capsule, the piriformis, and the superior portion of the conjoined tendon to 60 patients who underwent THA using the mini-incision posterolateral (PL) approach. The patients who had the DA approach had faster return to function in the early postoperative period compared to the PL group, consistent with other reports on the DA approach that do not explicitly describe these tendon releases. [13] This suggests that the DA approach's preservation of the SERs may not be the main factor contributing to accelerated recovery, [24] but may instead be the preservation of the ITB.

The DA approach is not without risk of intraoperative and postoperative complications, especially in the operations performed in the learning curve period. $[\underline{25}, \underline{26}]$ While relatively rare, the most common complications include intraoperative femoral fracture or perforation, acetabular fracture, lateral femoral cutaneous nerve (LFCN) palsy, and dislocation. Ankle fracture has also been reported with the use of traction tables for positioning. [27] Because of the location of the incision, superficial wound complications are also an issue, especially in patients with higher BMI. [28] These complications are more common (as high as 9-13\%) [29] in the first 50-100 cases, designated as the learning curve, $[\underline{26}, \underline{29}-\underline{30}]$ with early revision rates as high as 6\%. [30] These complication rates appear to decrease according to surgeon experience, with complication risk profiles similar to that of other hip approaches after the learning curve. $[\underline{21}, \underline{31}, \underline{32}]$ Additionally, risk of complications and expertise required both increase when using the DA approach for complex or revision cases, although it can be successfully performed. [33] The risk of an increased rate of these complications during the learning curve as well as the decreased extensibility of the approach remain deterrents for some surgeons to pursue the potential improved short term outcomes afforded by the approach, especially when long term outcomes have not been proven to be significantly better. $[\underline{17}, \underline{\underline{4}}, \underline{\underline{5}}]$

\section{SuperCap $^{\circledR}$ / Direct Superior Approach ${ }^{\circledR}$}

In 2002, Dr. Stephen Murphy first described the Supercapsular THA (SuperCap ${ }^{\circledR}$ Microport Orthopedics Inc., Arlington, TN, USA). This single-incision technique shifts the traditional posterior superficial dissection slightly proximal, preserving the ITB at the greater trochanter (FIGURE 3). Other modifications have been introduced (Direct Superior ${ }^{\circledR}$ Stryker Inc., Kalamazoo, MI, USA), but share in common the use of a superior capsulotomy and specialized instrumentation in an effort to avoid releasing the SER tendons.

Patients are placed in the lateral decubitus position on a standard operating table. The operative leg is placed in flexion, internal rotation, and adduction by placing the foot on a Mayo stand. The incision is typically in line with the femoral shaft just superior to the greater trochanter, extend-

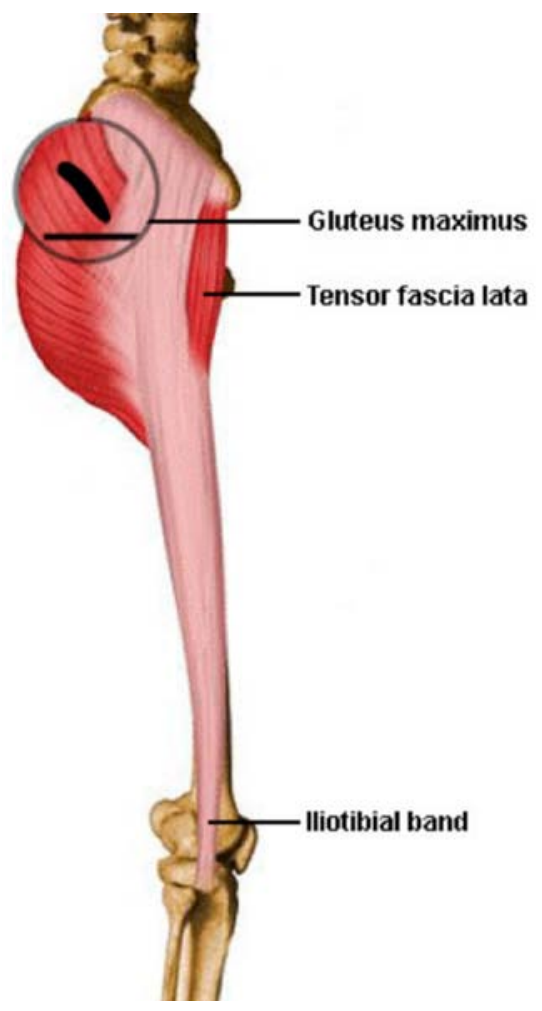

Figure 3: ITB-Sparing techniques: all superficial dissections do not violate ITB tendon. Image courtesy dept. washington.edu ing proximally be-

tween 6-8 $\mathrm{cm}$. Blunt dissection is performed to split the gluteus maximus in line with its fibers until the interval of the posterior border of the gluteus medius and the piriformis is identified. The piriformis and conjoined tendon attachments are preserved, if possible. Alternatively, the Direct Superior ${ }^{\circledR}$ or Northern approach uses the traditional PL approach deep interval (between the gluteus medius and the conjoined tendon with release of the piriformis and superior SERs) with the same ITB-sparing superficial dissection. [36] The gluteus minimus is reflected anteriorly from the superior capsule and the capsule incised along the superior aspect of the femoral neck, extending to the acetabulum.

Retractors placed along the anterior and posterior femoral neck allow complete visualization of the proximal femur. The proximal femur is prepared in line with the femoral shaft with the femoral head and neck in situ. This 
theoretically distributes stresses during femoral preparation, decreasing the risk of intraoperative femur fractures, and avoids overstretching the attached short external rotators that occurs with surgical dislocation. Acetabular preparation is performed using specialized instrumentation in the form of angulated reamers (FIGURE 4) and offset cup

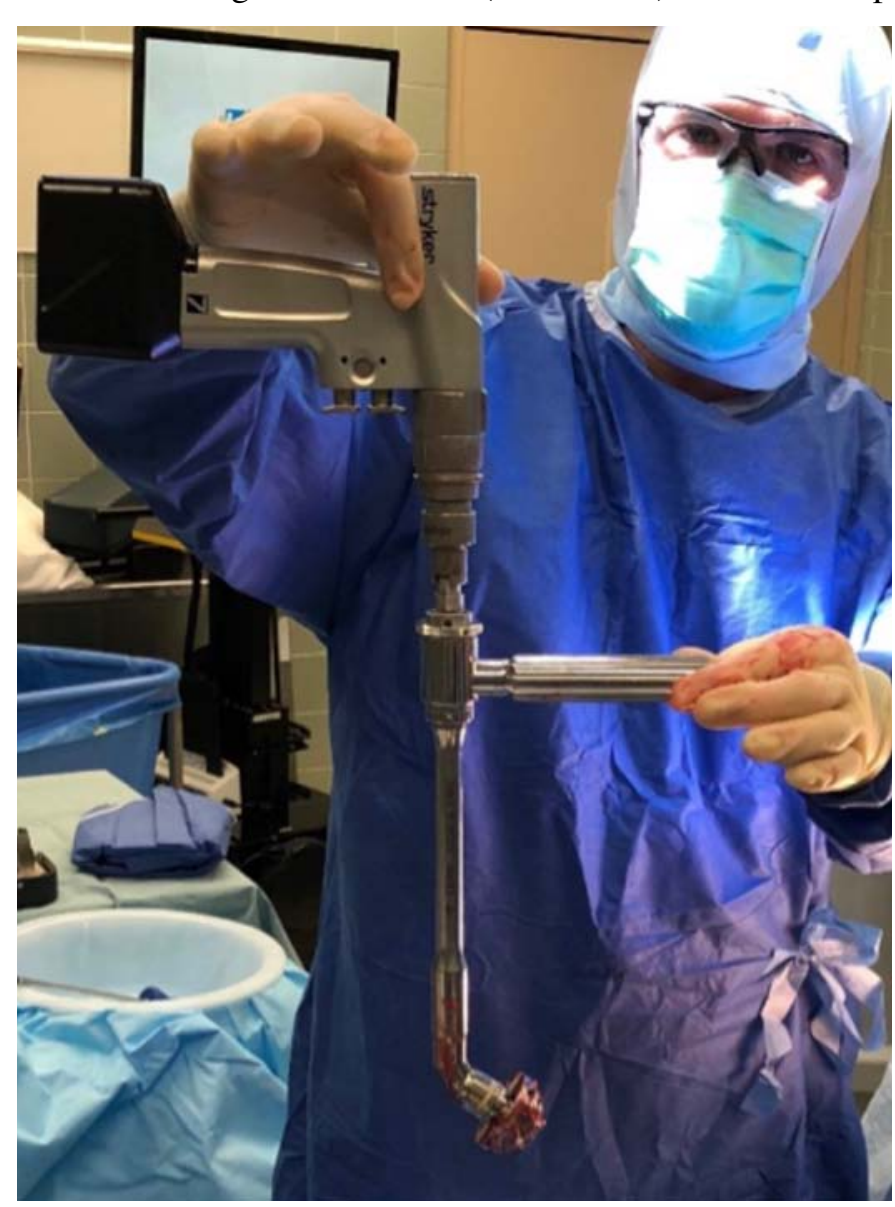

Figure 4: Angled reamer used to avoid violation of ITB tendon. Image courtesy Dr. Brandon Gough

inserters, with or without the use of CT guided navigation to aid in positioning. While these instruments are required for preparation of the bony structures, any implant system can be inserted during the procedure. The capsulotomy is closed in a normal fashion after trialing, implantation and in-situ reduction, and the piriformis insertion, if released, is repaired. Closure of the remaining layers is performed in the standard fashion. [37]

The tissue-preserving nature of the superior approach affords the stability of the traditional transgluteal approach while decreasing the potential risk of gait abnormalities and pain associated with the traditional posterior approach, presumably by preserving the ITB. This allows patients to routinely be placed into an accelerated rehabilitation protocol, permitting them to bear weight almost immediately after surgery without traditional hip precautions. [37, $\underline{38}]$
In a study of 218 consecutive patients undergoing total hip arthroplasty using this technique in 2010-2011, 87\% of patients were discharged within 2 days of surgery, and $99 \%$ of all patients were discharged home, with only 1 readmission for a GI bleed related to use of NSAIDs postoperatively. There was only 1 reoperation at 8 months postoperatively, an irrigation and debridement for continued pain with no evidence of infection. [38] Capuano reported on 463 patients including 275 primary elective THA and 188 femoral neck fracture patients that underwent THA using this technique. 375 patients $(75 \%)$ could walk with full weight bearing on the operative leg within 6 hours of the procedure. There was a $1 \%$ complication rate (1 dislocation, 2 malpositioned cups, 2 loosened cups), all within the first 20 cases. There were no further complications reported after the first 20 cases. [39]

\section{PATH $^{\circledR}$}

First reported by Dr. Penenberg in 2004, $\mathrm{PATH}^{\circledR}$ (Microport Orthopedics Inc., Arlington, TN, USA) utilizes the deep interval of the traditional PL approach, but differs from the traditional approach by shifting the superficial dissection superiorly to avoid disruption of the ITB at the greater trochanter. Rather than using an angulated reamer of SuperCap ${ }^{\circledR} /$ Direct Superior ${ }^{\circledR}$, acetabular reaming is accomplished by inserting a cannula through a portal in the soft tissues just posterior to the proximal femoral shaft, avoiding the ITB at the trochanter. This allows for direct visualization of the acetabulum during reaming, as well as the use of straight reaming handles for more familiar reaming control.

The patient is placed in the lateral decubitus position on a peg board, anterior on the table with the foot on a Mayo stand to allow for flexion, adduction and internal rotation of the leg. The incision starts at the proximal edge of the greater trochanter and extends proximally, angled 3045 degrees posteriorly. After incision of the overlying fascia, the gluteus maximus is bluntly dissected in line with its fibers. The interval is developed between the gluteus medius and the conjoined tendon. The piriformis insertion is identified and it is released along it's footprint, preserving as much length as possible for later repair. If necessary, the superior portion of the conjoined tendon may also be released for increased exposure. A posterior capsulotomy is performed, in line with the femoral neck, to the level of the acetabular rim before it is curved anteriorly along the posterior wall toward the lateral rim, creating a "J-shaped" capsulotomy. In the originally described approach, the hip was dislocated to allow for the femoral neck cut. However, 
in recent years, modifications (PATH II) allow for the femoral neck cut to be performed in-situ. Intracapsular Hohmann retractors are placed anterior and posterior to the femoral neck cut to expose the osteotomy site and protect soft tissues. A napkin ring of bone is typically removed as proximally on the neck as possible to facilitate head extrication, or, depending on surgeon preference, a single osteotomy with en bloc extrication may be performed. With a more posterior capsulotomy, a small (3-4mm) corner of the posterolateral greater trochanter without muscular attachment may impede the femoral neck osteotomy at the appropriate angle. This small piece of bone may be removed via cheilectomy to facilitate the osteotomy.

The hip is then slightly flexed and maximally internally rotated and the femur is prepped and broached. Once broaching to the appropriately sized implant is completed, a "cleanup" neck cut is performed using the inserted broach as a cutting guide. The appropriate depth and angle of the neck cut is determined by referencing the distance between the shoulder of the broach and the tip of the greater trochanter and comparing to preoperative templating. This allows for reliable and reproducible implant positioning.

The leg is then returned to the starting position for acetabular preparation. Large Hohmann acetabular retractors are used to provide visualization. A cannula is introduced through a downstream portal using an over-the-top guide, using the native acetabulum to direct placement. The cannula is directed through the soft tissues posterior to the femur and towards the acetabulum. Once the cannula is in place, an $8 \mathrm{~mm}$ reamer driver is inserted into the cannula and the reaming basket inserted and assembled in situ through the main exposure to perform reaming under direct visualization (FIGURES 5,6). The acetabular component is then inserted and impacted into a position of stability, with full visualization of the bony rim and cup during the insertion. Trialing is completed, intraoperative $\mathrm{x}$-rays may be taken, range of motion and stability is tested, and final implants are inserted. The posterior capsule, piriformis and conjoined tendons are then repaired and the incision closed in the standard fashion. [40]

Penenberg reported on 250 consecutive patients, without excluding patients for BMI or complexity, and followed them for two years. All patients were placed into an accelerated rehabilitation protocol, and $83 \%$ of patients were using a cane or no assistive device for ambulation at discharge. There were no dislocations, infections, or deep vein thrombosis in the group. Femoral and acetabular components were within the accepted limits in $96 \%$ and $97 \%$ of patients, respectively. [40]

Rasuli and Grofton analyzed the learning curve of the

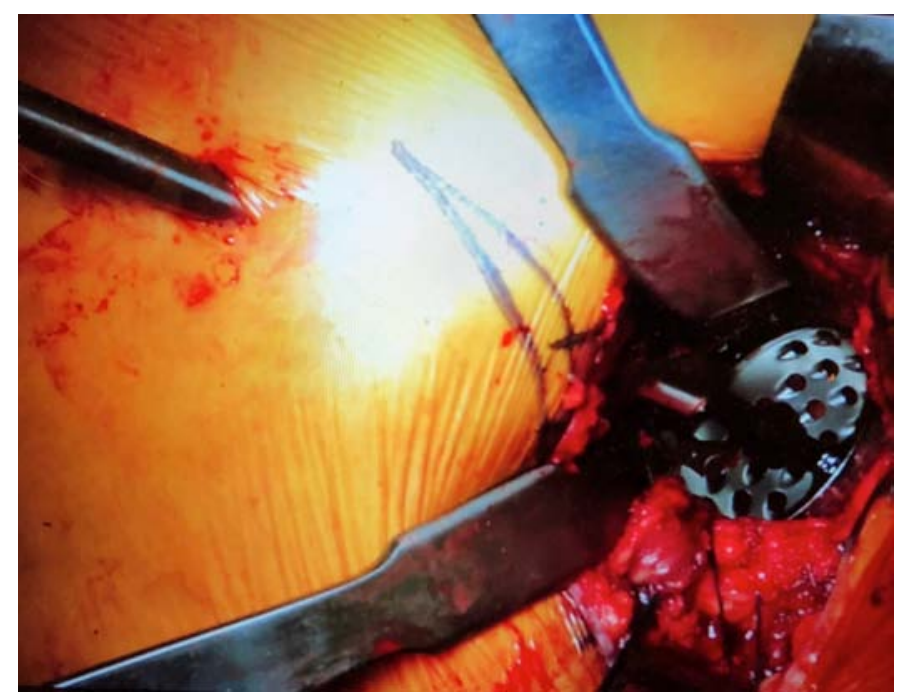

Figure 5: Small posterior incision and accessory portal with cannula in place allows for reaming and impacting cup without violation of ITB

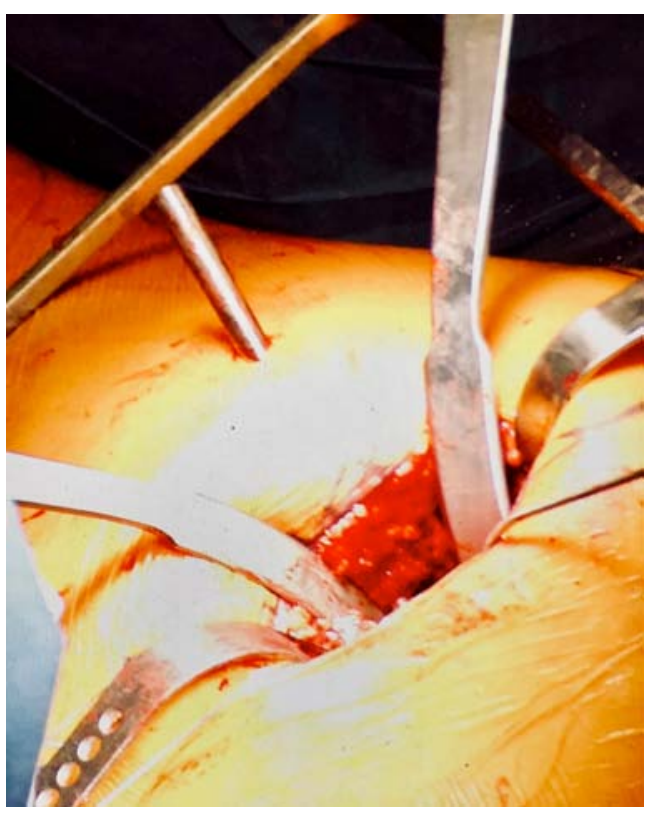

Figure 6:

Cannula provides excellent inline access to reamer basket without violating ITB and with minimal release of piriformis and external rotators

PATH procedure, showing reproducible acetabular anteversion and abduction (13.1 +/- 7.1 degrees, $42.9+/-7.6$ degrees), mean operative time of 114 minutes, and 2 complications in the first 50 cases (intraoperative femoral fracture in case 10 and a posterior dislocation at 6 weeks in case 26). [41] This relatively short learning curve demonstrates the safety of the procedure, likely due to the familiarity of the dissection to trained surgeons, while still affording the benefit of accelerated recovery after surgery. In addition, the incision and intervals are extensile and easily convertible to the standard posterior approaches.

\section{SuperPATH $^{\circledR}$}

Developed by Dr. James Chow, the SuperPATH ${ }^{\circledR}$ (Mi- 
croport Orthopedics Inc., Arlington, TN, USA) approach is a combination of certain aspects of the SuperCap/Direct Superior ${ }^{\circledR}$ and $\mathrm{PATH}^{\circledR}$ techniques, using the interval, capsulotomy, and femoral preparation of the Superior approach, but using the cannulated portal and straight acetabular reaming instruments that $\mathrm{PATH}^{\circledR}$ introduced. [41] The patients are similarly placed in the lateral decubitus position on a peg board, with the patient anterior on the bed to allow for a flexed, adducted, internally rotated position of the operative leg. The incision and dissection of SuperPATH $^{\circledR}$ are similar to SuperCap ${ }^{\circledR}$, with the interval between the gluteus medius and the piriformis being developed and attempted preservation of the piriformis and conjoined tendon insertions. After the capsulotomy is performed in approximately the 11:00 position, the femur is prepared in situ by "canoeing" a space into the femoral neck to allow for subsequent broach placement. The final broach is left in place for the femoral neck cut, similar to the technique described in $\mathrm{PATH}^{\circledR}$, using the distance between the broach shoulder and the tip of the greater trochanter as a reference. The head is removed en bloc. The acetabulum is then prepared in a similar fashion to that described above in the PATH $^{\circledR}$ approach. Once trialing is completed and final implants are placed the hip is reduced. Stability and range of motion is tested, and the capsule and soft tissues are repaired before standard closure is performed. [42]

This approach was developed to allow for the perceived advantages of the each of its predecessors, preserving the iliotibial band and external rotators, with in-line axial femoral preparation prior to the femoral neck cut as in the $\mathrm{Su}$ perCap $^{\circledR}$ approach and allowing for consistent acetabular reaming through the accessory portal as in the $\mathrm{PATH}^{\circledR}$ approach.

Results of the combined technique are similar to the other "micro" approaches. Chow et al reported on the first 110 patients, including the learning curve, and all patients had well placed femoral and acetabular components at 2 year follow-up, with an average hospital stay of 1.7 days. All patients were placed in a comprehensive management program including preoperative medical optimization and counseling, as well as accelerated rehabilitation postoperatively. Of note, no IV narcotics were required or used in the postoperative period. Chow also reported 4 complications related to the surgery in the first 330 patients $(1.2 \%)$ to undergo the operation, with 2 calcar fractures, 1 nondisplaced acetabular fracture, and 1 failure of cup fixation. [43,44]

Gofton et al reviewed 479 SuperPATH $^{\circledR}$ patients across 3 centers and evaluated readmission and discharge disposition rates. There was an all-cause readmission rate of $2.3 \%$ after an average LOS of 1.6 days. $91 \%$ of patients were discharged home routinely, $3.8 \%$ home with home health, and a combined $4.7 \%$ to skilled nursing and acute inpatient rehab facilities ( $4.1 \%$ and $0.6 \%$, respectively). [41] Gofton then analyzed the cost reduction using these results compared to the patients receiving a THA via a lateral approach by another surgeon at the same institution and found a $28.4 \%$ cost reduction in favor of SuperPATH. [45]

\section{Discussion}

Overall, hip arthroplasty is a surgical procedure with excellent long term outcomes, no matter the approach, so long as the components are well-placed and well-seated. Each traditional approach has its pros and cons, which have been extensively studied and improved upon with better techniques and technology over the last several decades. Because of the consistent long-term results, increasing attention has been placed on short term recovery with accelerated rehabilitation programs, multimodal pain management, and tissue preserving approaches. Results of early attempts at minimally invasive THA were generally unacceptable; however, continued innovation and exploration of tissue-preserving intervals, including the ones discussed in this review, have resulted in promising short-term outcomes with consistent medium and long-term results.

It was previously thought that the DA approach showed accelerated recovery due to sparing the SER tendons in comparison to the traditional posterolateral approach. However, recent descriptions of the technique using conjoined tendon releases without compromises in outcomes [24] suggest that the SER preservation may not be the true source of improved postoperative pain. One possible explanation for the shorter recovery may be that the DA approach spares the proximal ITB at the greater trochanter. The ITB-sparing approaches described above, while differing slightly in dissection planes, preservation of SER attachments, and instrumentation, share the preservation of the ITB in common with the DA approach. The evidence showing that early postoperative pain and function are similar between the approaches suggests that the ITB preservation may be the key common factor.

Penenberg proposes that preservation of the ITB in this area preserves the gluteus maximus attachment at the ITB, and that the sparing of this attachment is what contributes to decreased pain and improved function in the early postoperative period (personal communication). This sparing of the ITB makes these approaches distinctly different from traditional posterior and mini-posterior approaches, as neither truly spares this portion of the ITB and the gluteus maximus insertion. The authors of this review concur with this hypothesis, although further biomechanical study 
is needed to further define the role this structure plays.

When all is said and done, there is no conclusive evidence that one approach to THA is superior to another. The above-mentioned techniques offer different options to perform this common procedure in a manner that preserves the ITB and the insertion of the superior gluteus maximus. While the anterior approach is much more extensively studied, the learning curve and potential complications reported in the literature may deter surgeons from adopting the technique into practice. The other techniques mentioned here offer an alternative to the DA approach with promising short and medium-term results reported in the literature.

The decision on which approach to use in daily practice should be a function of the surgeon's training and experience. That said, the senior author of this review utilizes the PATH approach for the following reasons: 1) the ITB and gluteus maximus tendon sparing nature of the approach, which appears to influence early postoperative pain and function, 2) the small learning curve, likely due to the familiarity of the anatomy, approach, and orientation from a previously-used posterior approach, 3 ) the ease of use of the instrumentation introduced by this technique, 4) the extensile nature of the approach, should the need arise, allowing standard posterior approach access to the femur and acetabulum, and 5) the avoidance of common complications of the DA approach, such as LFCN injury, femoral perforation during broaching, and wound infections due to the incision being made in the groin area.

We recognize the need for increased clinical data to further demonstrate the safety and efficacy of these relatively new techniques. Longer term data is currently being collected. Further reporting of these results is warranted and merits discussion in the future.

\section{References}

1. Chechik O, Khashan M, Lador R, Salai M, Amar E. Surgical approach and prosthesis fixation in hip arthroplasty world wide. Arch Orthop Trauma Surg. 2013;133(11):1595-1600.

2. Huang BK, Campos JC, Michael Peschka PG, et al. Injury of the gluteal aponeurotic fascia and proximal iliotibial band: anatomy, pathologic conditions, and MR imaging. Radiographics. 2013 Sep-Oct;33(5):1437-52.

3. Evans P. The postural function of the iliotibial tract. Ann $R$ Coll Surg Engl. 1979;61(4):271-280.

4. Flato R, Passanante GJ, Skalski MR, Patel DB, White EA, Matcuk GR. The iliotibial tract: imaging, anatomy, injuries, and other pathology. Skeletal Radiol. 2017;46(5):605-622.

5. Fairclough J, Hayashi K, Toumi H, et al. The functional anatomy of the iliotibial band during flexion and extension of the knee: implications for understanding iliotibial band syndrome. J Anat. 2006;208(3):309-316. doi:10.1111/j.14697580.2006.00531.x.

6. Inman, V. Functional Aspects of the Abductor Muscles of the Hip. J Bone Jt Surgery-American Vol. 29(3):607-619, July 1947.

7. Müller M, Schwachmeyer V, Tohtz S, et al. The direct lateral approach: Impact on gait patterns, foot progression angle and pain in comparison with a minimally invasive anterolateral approach. Arch Orthop Trauma Surg. 2012;132(5):725-731.

8. Rachbauer F, Kain MSH, Leunig M. The History of the Anterior Approach to the Hip. Orthop Clin North Am. 2009;40(3):311-320.
9. Smith-Petersen MN: A new supra-articular subperiosteal approach to the hip joint. Am J Orthop Surg (Phila Pa) 1917;15:593.

10. Light TR, Keggi KJ: Anterior approach to hip arthroplasty. Clin Orthop Relat Res 1980;152:255-260.

11. Post ZD, Orozco F, Diaz-Ledezma C, et al. Direct Anterior Approach for Total Hip Arthroplasty: Indications, Technique, and Results. J Am Acad Orthop Surg. 2014;22(9):595-603.

12. Leunig M, Faas M, von Knoch F, et al. Skin crease 'bikini' incision for anterior approach total hip arthroplasty: surgical technique and preliminary results. Clin $\mathrm{Or}$ thop Relat Res. 2013 Jul;471(7):2245-52.

13. Rodriguez JA, Cooper HJ, Robinson J. Direct anterior approach to THR: what it is and what it is not. Curr Rev Musculoskelet Med. 2013 Dec;6(4):276-8.

14. Meneghini RM, Pagnano MW, Trousdale RT, Hozack WJ. Muscle damage during MIS total hip arthroplasty: Smith-Petersen versus posterior approach. Clin Orthop Relat Res. 2006 Dec;453:293-8

15. Barrett WP, Turner SE, Leopold JP. Prospective randomized study of direct anterior vs postero-lateral approach for total hip arthroplasty. J Arthroplasty. 2013;28(9):1634-1638.

16. Restrepo C, Parvizi J, Pour AE, et al. Prospective Randomized Study of Two Surgical Approaches for Total Hip Arthroplasty. J Arthroplasty. 2010;25(5):671-679.

17. Berend KR, Lombardi A V, Seng BE, et al. Enhanced Early Outcomes with the Anterior Supine Intermuscular Approach in Primary Total Hip Arthroplasty. J Bone Jt Surgery-American Vol. 2009;91(Suppl 6):107-120.

18. Martin CT, Pugely AJ, Gao Y, et al. A comparison of hospital length of stay and short-term morbidity between the anterior and the posterior approaches to total hip arthroplasty. J Arthroplasty. 2013;28(5):849-854

19. Meermans G, Konan S, Das R, et al. The direct anterior approach in total hip arthroplasty. Bone Joint J. 2017;99-B(6):732-740.

20. Yue C, Kang P, Pei F. Comparison of Direct Anterior and Lateral Approaches in Total Hip Arthroplasty: A Systematic Review and Meta-Analysis (PRISMA) Medicine (Baltimore). 2015;94(50):e2126.

21. Zhao H-Y, Kang P-D, Xia Y-Y, Shi X-J, Nie Y, Pei F-X. Comparison of Early Functional Recovery After Total Hip Arthroplasty Using a Direct Anterior or Posterolateral Approach: A Randomized Controlled Trial. J Arthroplasty. 2017.

22. Schweppe ML, Seyler TM, Plate JF, Swenson RD, Lang JE. Does surgical approach in total hip arthroplasty affect rehabilitation, discharge disposition, and readmission rate? Surg Technol Int. 2013;23:219-227.

23. Poehling-Monaghan KL, Kamath AF, Taunton MJ, Pagnano MW. Direct Anterior versus Miniposterior THA With the Same Advanced Perioperative Protocols: Surprising Early Clinical Results. Clin Orthop Relat Res. 2014;473(2):623-631.

24. Rodriguez JA, et al. Does the direct anterior approach in THA offer faster rehabilitation and comparable safety to the posterior approach? Clin Orthop Relat Res. 2014 Feb;472(2):455-63.

25. Barton C, Kim PR. Complications of the direct anterior approach for total hip arthroplasty. Orthop Clin North Am. 2009;40(3):371-375.

26. Masonis J, Thompson C, Odum S. Safe and accurate: learning the direct anterior total hip arthroplasty. Orthopedics. 2008;31(12 Suppl 2).

27. Matta JM, Shahrdar C, Ferguson T. Single-incision Anterior Approach for Total Hip Arthroplasty on an Orthopaedic Table. Clin Orthop Relat Res. 2005;441(NA;):115-124.

28. Purcell RL, Parks NL, Gargiulo JM, Hamilton WG. Severely Obese Patients Have a Higher Risk of Infection Following Direct Anterior Approach Total Hip Arthroplasty. J Arthroplasty. 2016:2-5.

29. Woolson ST, Pouliot MA, Huddleston JI. Primary Total Hip Arthroplasty Using an Anterior Approach and a Fracture Table. Short-term Results From a Community Hospital. J Arthroplasty. 2009;24(7):999-1005.

30. de Steiger RN, Lorimer M, Solomon M. What Is the Learning Curve for the Anterior Approach for Total Hip Arthroplasty? Clin Orthop Relat Res. 2015;473(12):3860-3866.

31. Barnett SL, Peters DJ, Hamilton WG, Ziran NM, Gorab RS, Matta JM. Is the Anterior Approach Safe? Early Complication Rate Associated With 5090 Consecutive Primary Total Hip Arthroplasty Procedures Performed Using the Anterior Approach. J Arthroplasty. 2016;31(10):2291-2294.

32. Jewett BA, Collis DK. High complication rate with anterior total hip arthroplasties on a fracture table. Clin Orthop Relat Res. 2011;469(2):503-507.

33. Molenaers B, Driesen R, Molenaers G, Corten K. The Direct Anterior Approach for Complex Primary Total Hip Arthroplasty: The Extensile Acetabular Approach on a Regular Operating Room Table. J Arthroplasty. 2017;32(5):1553-1559.

34. Mirza AJ, Lombardi A V., Morris MJ, Berend KR. A mini-anterior approach to the hip for total joint replacement: Optimising results: Improving hip joint replacement outcomes. Bone Jt J. 2014;96B(11):32-35.

35. Sendtner E, Borowiak K, Schuster T, Woerner M, Grifka J, Renkawitz T. Tackling the learning curve: Comparison between the anterior, minimally invasive (Micro- 
hip $^{\circledR}$ ) and the lateral, transgluteal (Bauer) approach for primary total hip replacement. Arch Orthop Trauma Surg. 2011;131(5):597-602.

36. Amanatullah DF, Masini MA, Roger DJ, Pagnano MW. Greater inadvertent muscle damage in direct anterior approach when compared with the direct superior approach for total hip arthroplasty. Bone Joint J. 2016 Aug;98-B(8):1036-42.

37. Murphy SB, Ecker TM. THA Performed using Conventional and Navigated Tissue-preserving Techniques. 2006;(453):160-167.

38. Wellman SS, Murphy AC, Gulcynski D, Murphy SB. Implementation of an accelerated mobilization protocol following primary total hip arthroplasty: Impact on length of stay and disposition. Curr Rev Musculoskelet Med. 2011;4(3):84-90.

39. Capuano N, Del Buono A, Maffulli N. Tissue preserving total hip arthroplasty using superior capsulotomy. Oper Orthop Traumatol. 2015;27(4):334-341.

40. Penenberg BL, Bolling WS, Riley M. Percutaneously assisted total hip arthroplasty (PATH): a preliminary report. J Bone Joint Surg Am. 2008 Nov;90 Suppl 4:209-20.

41. Rasuli KJ, Gofton W. Percutaneously assisted total hip (PATH) and Supercapsular percutaneously assisted total hip (SuperPATH) arthroplasty: learning curves and early outcomes. Ann Transl Med. 2015;3(13):179.

42. Chow JC, Della Torre PK, Fitch DA. SuperPATH and Micro-superior Total Hip Arthroplasty. In: Scuderi GR, Tria AJ, eds. Minimally Invasive Surgery in Orthopedics. Cham: Springer International Publishing; 2016:541-552.

43. Della Torre PK, Fitch DA, Chow JC. Supercapsular percutaneously-assisted total hip arthroplasty: radiographic outcomes and surgical technique. Ann Transl Med. 2015;3(13):180.

44. Chow J, Penenberg B, Murphy S. Modified micro-superior percutaneously-assisted total hip: Early experiences \& case reports. Curr Rev Musculoskelet Med. 2011;4(3):146-150.

45. Gofton W, Fitch DA. In-hospital cost comparison between the standard lateral and supercapsular percutaneously-assisted total hip surgical techniques for total hip replacement. Int Orthop. 2016;40(3):481-485.
SUBMISSION HISTORY

Submitted August 9, 2018

Reviewed December 12, 2018

Accepted December 17, 2018

Published December 21, 2018

\section{AUTHOR AFFILIATIONS}

1 Russell Nevins, MD

Nevada Orthopedic \& Spine Center, 7455 W. Washington Avenue, Ste. 160, Las Vegas, NV 89128

2 Kevin M Sagers, DO

Valley Hospital Medical Center, 620 Shadow Ln Las Vegas, NV 89106

(Direct inquires to Russell Nevins, russellnevinsmd@cox.net)

\section{AUTHOR DISCLOSURES}

The authors declare that there are no disclosures regarding the publication of this paper.

\section{COPYRIGHT \& OPEN ACCESS}

C) 2018 Nevins, Sagers. All rights reserved.

Authors retain copyright and grant the journal right of first publication with the work. Reconstructive Review is an open access publication and follows the Creative Commons Attribution-NonCommercial CC BY-NC. This

license allows anyone to download works, build upon the material, and share them with others for non-commercial purposes as long as they credit the senior author, Reconstructive Review, and the Joint Implant Surgery \& Research Foundation (JISRF). An example credit would be: "Courtesy of (senior author's name), Reconstructive Review, JISRF, Chagrin Falls, Ohio". 